\title{
Palatal rugae patterns as an adjunct in gender identification
}

\author{
Kunal Kumar ${ }^{1}$, Shivendra Choudhary ${ }^{2 *}$, Revati Singh ${ }^{3}$, Neal Bharat Kedia ${ }^{4}$, Abhinav Kumar Singh ${ }^{5}$, \\ Ravi Shanker Prasad ${ }^{6}$
}

${ }^{1,3}$ Senior Resident, ${ }^{2}$ Associate Professor and Head, ${ }^{4}$ Professor, ${ }^{5}$ Reader, ${ }^{6}$ Tutor, Dept. of Dentistry, ${ }^{\mathbf{1 - 3}}$ Patna Medical College and Hospital, Patna, Bihar, ${ }^{4,5}$ Buddha Institute of Dental Sciences \& Hospital, Patna, Bihar, ${ }^{6}$ Darbhanga Medical College, Darbhanga, Bihar, India

\author{
*Corresponding Author: Shivendra Choudhary
}

Email: sky20083@gmail.com

\begin{abstract}
Palatal rugae pattern of an individual may be considered as a practical adjunct for gender determination in identification of an individual for forensic medicine. Palatal rugae possess distinct traits of an ideal forensic parameter like uniqueness, postmortem resistance, and stability. The present study describes sexual dimorphism using palatal rugae pattern. A total number of 200 subjects in the age range of 17 to 25 years were studied with the help of study casts for palatal rugae pattern. Sexual dimorphism was assessed using statistical analysis. The p-value was calculated using Chi- square test. Results showed prominent converging rugae pattern in females as compared to males which showed prominence of curved rugae pattern.
\end{abstract}

Keywords: Dimorphism, Forensic, Palatal rugae.

\section{Introduction}

Establishment of identity of anonymous individual is the main aim of forensic medicine, which also supplement the law enforcement agencies. ${ }^{1}$ Most challenging situations in forensics is mass disasters where severely decomposed and fragmented bodies are the only left over for the identification of unknown. ${ }^{2}$ In this context, craniofacial structures being relatively resistant to decay with standard and well defined anatomical landmarks which are easy to locate. ${ }^{3}$ Among them, palatal rugae are commonly protected due to its anatomic position. Therefore, the stability and uniqueness of the palatal rugae pattern, it has been considered as one of the relevant parameters for human identification in the field of forensic medicine. The study of palatal rugae pattern for human identification referred as Rugoscopy. ${ }^{4}$ The pattern of palatine rugae exhibits gender variations $^{5}$ and hence, careful study of these can enable reliable determination of unknown person in life, particularly when other preferred predictors are destroyed or fragmented.

The present study was therefore, ventured to explore the role of palatal rugae pattern in sexual dimorphism which can be used as an adjunct with other parameters for person identification in forensic medicine.

\section{Materials and Methods}

A total number of 200 subjects (100 male and 100 female) were recruited from patients attending the outpatient
Department of Dentistry of Patna Medical College \& Hospital. The study was conducted on the patients in the age range 17 to 25 years. All the participants were of Indian origin. Thorough clinical examination was performed, patients were informed about the study, and a written consent was obtained. Impressions of maxillary arches were made with the alginate material (normal set type), and were poured immediately with type II dental stone so as to minimize the dimensional changes in the study casts. The first investigator made the impressions and poured the casts. The second investigator (Chief investigator) was blinded for the study group. Rugae patterns on every cast were delineated using a sharp graphite pencil under adequate light and magnification and classified according to the Thomas and Kotze's classification. Palatal rugae pattern were identified on the casts. The findings were subjected to statistical analysis and the results were obtained in percentage and p- value was calculated using Chi- square test.

\section{Results}

Rugae patterns were identified as branched, converge, curve and straight. Male showed prominence of curved type (44\%) whereas females showed converged rugae pattern in abundance $(70 \%)$. P-value using Chi- square test was calculated which showed statistical significant differences among converged and curved rugae pattern between males and females.

Table 1: Different rugae pattern in male and female

\begin{tabular}{|l|c|c|c|c|}
\hline \multirow{2}{*}{ Rugae pattern } & & \multicolumn{2}{|c|}{ Gender } & \multirow{2}{*}{ Total } \\
\cline { 2 - 5 } & & Male & Female & 34 \\
\hline \multirow{2}{*}{ Converge } & Count & 14 & 20 & $17.0 \%$ \\
\cline { 2 - 5 } & Percentage & $14.0 \%$ & $20.0 \%$ & 90 \\
\cline { 2 - 5 } & Count & 70 & 20 & $45.0 \%$ \\
\hline
\end{tabular}




\begin{tabular}{|l|c|c|c|c|}
\hline & Percentage & $16.0 \%$ & $\mathbf{4 4 . 0 \%}$ & $30.0 \%$ \\
\hline \multirow{2}{*}{ Straight } & Count & 0 & 16 & 16 \\
\cline { 2 - 5 } & Percentage & $0 \%$ & $16.0 \%$ & $8.0 \%$ \\
\hline \multirow{2}{*}{ Total } & Count & 100 & 100 & 200 \\
\cline { 2 - 5 } & Percentage & $100.0 \%$ & $100.0 \%$ & $100.0 \%$ \\
\hline
\end{tabular}

Table 2: $p$-value of different rugae pattern

\begin{tabular}{|l|c|c|}
\hline Rugae pattern & & Gender \\
\hline \multirow{4}{*}{ Branched } & Chi-Square (a,b,c) & .529 \\
\cline { 2 - 3 } & Df & 1 \\
\cline { 2 - 3 } & Asymp. Sig. & .467 \\
\cline { 2 - 3 } & Chi-Square (a,b,c) & 13.889 \\
\cline { 2 - 3 } & Df & 1 \\
\hline \multirow{3}{*}{ Curve } & Asymp. Sig. & .000 \\
\cline { 2 - 3 } & Chi-Square (a,b,c) & 6.533 \\
\cline { 2 - 3 } & Df & 1 \\
\cline { 2 - 3 } & Asymp. Sig. & .011 \\
\hline
\end{tabular}

conclude that curved pattern of rugae are prominent in male

\section{Discussion}

Sex determination is one of the prime factors employed to establish the identity of an individual. The accuracy of sex determination using diverse parameters of the body such as craniofacial morphology and measurement on the pubis ranges from $96 \%$ to $100 \% .^{6}$ Accurate sex assessment of skeletal remains has great importance in forensic and anthropological investigations. For optimal outcome, as many criteria as are available must be utilised. ${ }^{3,4}$

Palatal rugae, in addition to being unique to an individual, are protected from trauma by their internal position in the head and insulated from heat by the tongue and buccal fat pads, unlike other structures that can get destroyed. ${ }^{3,7}$

The present study describes sexual dimorphism using palatal rugae pattern. A total number of 200 subjects in the age range of 17 to 25 years were studied with the help of study casts for palatal rugae pattern. Sexual dimorphism using palatal rugae was assessed where rugae pattern showed highly significant p-value (.000) for converge type of rugae, while not significant for the other types. In the present study, converge type of rugae pattern was more common in females than males and curve type of rugae pattern was more common in males as compared to females. These results were in accordance of the studies done by Fahmi et al (2001) and Sharma P et al (2009). ${ }^{6,7}$ However Mahnaz et al (2017), could not differentiate males and females in Iranian population and had low abilities to classify the racial subsets. ${ }^{8}$ In contrast to our results Pereira et al (2018) showed more number of converging rugae in males and more of diverging patterns of rugae in females in Mumbai population. ${ }^{9}$ Dwievdi et al (2016) showed wavy rugae prominence in males and straight type in females. ${ }^{10}$

Differences in results of studies may be attributed to the ethnicity and origin of substance. More studies are needed to conclude palatal rugae pattern in gender determination with consideration of more aspects like religion and ethnicity. From the present study we can substantially and converged in females in population of Bihar. Hence, it could be a valuable diagnostic tool in forensic odontology.

\section{Conclusion}

The palate represents a suitable landmark for unique and identifying features with additional benefit of survival in most postmortem events that can disrupt or change other body parts. However, further studies are needed to investigate the possibility that there is a distinct ethnic difference in the palatal rugae morphology in human identification with large sample size.

\section{Source of funding}

None.

\section{Conflict of interest}

None.

\section{References}

1. Yadav AB, Yadav SK, Kedia NB, Singh AK. An Odontometric Approach for Estimation of Stature in Indians: Cross- Sectional Analysis. J Clinic Diag Res 2016;10(3): ZC24-6.

2. Gadicherla P, Saini D, Bhaskar M. Palatal rugae pattern: An aid for sex identification. J Forensic Dent Sci 2017;9(1):48.

3. Yadav AB, Kale AD, Mane DR, Yadav SK, Hallikerimath S. Stature estimation from regression analysis of facial anthropometry in Indian population. J Oral Maxillofac Pathol 2019;23:311.

4. Balgi P, Bhalekar B, Bhalerao K, Bhide E, Palaskar S, Kathuriya P. Study of palatal rugae pattern in gender identification. J Dent Allied Sci 2014;3:13-6.

5. Nayak P, Acharya AB, Padmini AT, Kaveri H. Differences in the palatal rugae shape in two populations of India. Arch Oral Biol 2007;52:977-82.

6. Fahmi, Faisal M, Sharmani A, Saleh M, Talic, Yousef F. Rugae pattern in a Saudi population sample of males and females. Saudi Dental J 2001; 13 (2):92-5.

7. Sharma P, Saxena S, Rathod V. Comparative reliability of cheiloscopy and palatoscopy in human identification. Indian $J$ Dent Res 2009;20(4):453-7. 
8. Sheikhi M, Zandi M, Ghazizadeh M. Assessment of palatal rugae pattern for sex and ethnicity identification in an Iranian population. Dent Res J 2018;15:50-6.

9. Pereira T, Shetty S, Surve R, Gotmare S, Kamath P, Kuamr S. Palatoscopy and odontometrics for sex identification and hereditary pattern analysis in a Navi Mumbai population: A cross sectional study. J Oral Maxillofac Pathol 2018;22:271-8.

10. Dwivedi N, Nagarajappa AK. Morphological analysis of palatal rugae pattern in central India population. J Int Soc Prevent Communit Dent 2016;6:417-22.

\footnotetext{
How to cite this article: Kumar K, Choudhary S, Singh R, Kedia NB, Singh AK, Prasad RS. Palatal rugae patterns as an adjunct in gender identification. Int $J$ Oral Health Dent 2019;5(4):195-7.
} 